\title{
Ethylmalonic Encephalopathy 1 Protein Is Increased in Colorectal Adenocarcinoma
}

\author{
EKIN OZLUK ${ }^{1}$, DOMENICO COPPOLA $^{2}$, ISLAM Z. MOHAMMAD $^{1}$, TARIF ISLAM $^{1}$, \\ GHALI GHALI ${ }^{3}$, CHRISTOPHER G. KEVIL $^{1}$ and RODNEY E. SHACKELFORD ${ }^{1}$ \\ ${ }^{1}$ Department of Pathology and Translational Pathobiology, LSU Health Sciences Center, Shreveport, LA, U.S.A; \\ ${ }^{2}$ Department of Anatomic Pathology, H. Lee Moffitt Cancer Center and Research Institute, Tampa, FL, U.S.A; \\ ${ }^{3} \mathrm{Head}$ and Neck Oncologic/Microvascular Reconstructive Surgery Department of Oral and Maxillofaciall \\ Head and Neck Surgery, Louisiana State University Health Sciences Center, Shreveport, LA, U.S.A.
}

\begin{abstract}
Background/Aim: Ethylmalonic encephalopathy 1 protein (ETHE1) plays an important role in sulfide catabolism and polysulfide formation. As sulfides and polysulfides have recently been identified as playing important roles in cancer, we hypothesized that ETHE1 expression would be increased in colon cancer. Materials and Methods: We used tissue microarray analysis to compare ETHE1 expression in benign colonic epithelium compared to colonic adenocarcinoma. In total, 26 benign colonic epithelial samples were compared to 122 cases of colonic adenocarcinomas. Results: Compared to benign colonic epithelium, ETHE1 expression was significantly increased ( two-fold) in colonic adenocarcinoma. Additionally, this expression increased with increasing colon cancer tumor grades. Conclusion: ETHE1 expression is increased in colon cancer compared to benign colonic epithelium. These data, combined with previous studies, suggest that ETHE1 may contribute to colon carcinogenesis by promoting tumor cell bioenergetics and polysulfide formation.
\end{abstract}

Ethylmalonic encephalopathy 1 protein (ETHE1), also known as sulfur dioxygenase, is located on chromosome $19 \mathrm{q} 13$ and encodes a mettallo $\beta$-lactamase family enzyme containing a mononuclear iron center (1-4). Loss-of-function ETHE1 mutations are associated with ethylmalonic encephalopathy,

This article is freely accessible online.

Correspondence to: Rodney E. Shackelford, Associate Professor of Pathology, Department of Pathology and Translational Pathobiology, LSU Health Sciences Center, Shreveport, LA, U.S.A. Tel: +1 3185256479, e-mail: rodney.shackelford@1suhs.edu

Key Words: Ethylmalonic encephalopathy 1 protein, ETHE1, colon cancer, polysulfides. a rare infantile autosomal recessive metabolic disorder affecting the brain, gastrointestinal tract, and peripheral vessels, characterized by psychomotor regression with generalized hypotonia, evolving into tetraparesis, dystonia, and subsequent global neurological failure and death in the first decade (3-5). Patients with ethylmalonic encephalopathy exhibit lactic acidemia, elevated urine ethylmalonic and methylsuccinic acids, and low skeletal muscle mitochondrial respiratory complex IV activity (3-5).

ETHE1 functions in the mitochondrial matrix oxidizing sulfides to sulfate or sulfate esters, and taurine, through the sequential actions of sulfide: quinone oxidoreductase, ETHE1, and rhodanese (6). Specifically, ETHE1 detoxifies/oxidizes hydrogen sulfide (H2S) and glutathione polysulfides (glutathione-persulfide, -trisulfide, and -tetrasulfide), but not cysteine- or homocysteine- persulfides/polysulfides (6-8). Interestingly, ETHE1 also exhibits cysteine protein polysulfidation activities. ETHE1 persulfide dioxygenase and polysulfidation activities are dependent on Cys247 polysulfidation and replacement of the Cys247 with serine ablates both enzymatic activities (8). The enzymes that synthesize $\mathrm{H} 2 \mathrm{~S}$, cystathionine $\beta$-synthase (CBS), cystathionine $\gamma$-lyase (CSE), and 3-mercaptopyruvate sulfurtransferase (3MST), also function in polysulfide synthesis; suggesting that these enzymes and ETHE1 function together to regulate cellular polysulfide levels (8-10).

Recently, one or more of the enzymes that synthesize H2S have been found to be increased in many different human malignancies, with one study of oral squamous cell carcinoma also showing significantly increased tumor $\mathrm{H} 2 \mathrm{~S}$ levels (11-13). Additionally, there is recent evidence that polysulfides also play a role in cancer promotion (12). Based on this, we hypothesized that ETHE1 may be increased in cancer. Herein we used tissue microarray analysis (TMA) to examine ETHE1 expression in benign colonic epithelium compared to colonic adenocarcinoma. 


\section{Materials and Methods}

Tissue microarray (TMA). The TMA CO1921 was purchased from US Biomax, Inc. (Rockville, MD, USA). The TMA contained 32 benign colon biopsies and 159 cases of colonic adenocarcinoma. The TMA was interrogated by an ETHE1 antibody. All tissue samples in the TMAs were $1.0 \mathrm{~mm}$ in diameter.

ETHE1 immunohistochemistry (IHC). The concentration of the primary ETHE1 antibody was optimized to normal kidney as a positive control tissue. The negative control was the same tissue interrogated without the primary ETHE1 antibody. The staining of the TMA was performed at the Tissue Core Histology Lab Facility at the Moffitt Cancer Center. The microarray slides were stained using a Ventana Discovery XT automated system (Ventana Medical Systems, Tucson, AZ, USA) as per the manufacturer's protocol with proprietary reagents. Briefly, the slide was deparaffinized on the automated system with EZ Prep solution (Ventana Medical Systems). The heat-induced antigen retrieval method was used in Cell Conditioning (Ventana Medical Systems). Rabbit monoclonal antibody to human ETHE1 (rabbit anti-ETHE1: EPR11697 ab174302, Abcam, Cambridge, MA) was used at a 1:1,000 concentration in Dako antibody diluent (Dako, Carpentaria, CA, USA) and incubated for $60 \mathrm{~min}$. Ventana anti-rabbit secondary antibodies were used for $16 \mathrm{~min}$. The detection system used was the Ventana OmniMap kit. Slides were then dehydrated and coverslipped per standard protocol.

Evaluation of staining. Relative ETHE1 protein expression was determined as average sample immunostain intensity scored on a 0 to 3 scale as follows: no staining: 0 , light staining: 1 , moderate staining: 2, and heavy staining: 3 . The percentage of cells stained was measured, with no detectable staining as $0,1-33 \%$ as $1,34-66 \%$ as 2 , and $67-100 \%$ as 3 . The final IHC score was the product of the percentage of cells stained multiplied by the intensity score, allowing for a minimal score of 0 and a maximal score of 9. Cytoplasmic ETHE1 staining was seen in all tissue samples examined, although at low to moderate levels in benign colonic epithelium. The cytoplasmic staining showed a punctate pattern, suggestive of a mitochondrial distribution. Based on this, ETHE1 staining in the cytoplasmic compartment was measured and quantified.

Statistical analysis of TMA results. The standard error of the mean (SEM) IHC score was calculated by using the standard deviation for the staining scores of each tumor type and dividing this number by the square root of the sample size (14).

\section{Results}

Following IHC processing, 6 of the 32 samples of benign colonic epithelium were lost. In the colonic adenocarcinoma samples, 37 were not counted, leaving 122 cases. Of those not counted, some were lost in processing, while cases of signet ring cell or mucinous adenocarcinomas, or colonic adenocarcinomas graded with more than one grade were also excluded from the study. Of the remaining colonic adenocarcinomas 19 were grade I, 89 were grade II, and 14 were grade III. The number of cases examined for ETHE1 staining, the quantified IHC results, and the SEM of each
Table I. Relative ethylmalonic encephalopathy 1 protein immunohistochemical staining in benign colonic epithelium and colonic adenocarcinoma. ETHE1, Ethylmalonic encephalopathy 1 protein; IHC, immunohistochemistry; SD, standard deviation; SEM, standard error to the mean.

\begin{tabular}{lcccc}
\hline Tissue & $\begin{array}{c}\text { Number of } \\
\text { cases }\end{array}$ & $\begin{array}{c}\text { ETHE1 } \\
\text { staining }\end{array}$ & SD & SEM \\
\hline Benign colon & 26 & 2.96 & 0.19 & 0.039 \\
All colonic adenocarcinomas & 122 & 5.67 & 2.02 & 0.17 \\
Grade I & 19 & 5.02 & 2.35 & 0.53 \\
Grade II & 89 & 5.49 & 1.80 & 0.39 \\
Grade III & 14 & 6.39 & 2.03 & 0.22 \\
\hline
\end{tabular}

data set are given in Table I. Examples of ETHE1 IHC are shown in Figure 1.

ETHE1 exhibited strong apical localization in benign colonic epithelium, with lower expression deeper in the colonic epithelia. Staining in the colonic adenocarcinoma was uniform and moderately increased compared to benign colonic adenocarcinoma, as previously found (15). Overall, $38.40 \%$ of the colonic adenocarcinoma samples scored 3 by the analysis employed here, $53.90 \%$ scored 6 , and $17.73 \%$ scored 9 . When ETHE1 expression in grades I-III adenocarcinoma was compared, a slight to moderate increase in expression was seen with increasing tumor grades (Table I and Figure 1).

\section{Discussion}

ETHE1 plays a significant role in $\mathrm{H} 2 \mathrm{~S}$ metabolism and protein polysulfide formation, suggesting that it might play a role in cancer (8-15). Previously, ETHE1 was found to be increased in the sera of early lung cancer patients by protein serologic array analyses of 352 lung cancer cases, compared to 93 healthy samples, and 101 patients with benign pulmonary lesions (16). Similarly, ETHE1 expression was increased in 6 of 6 colorectal cancer cell lines compared to a nonmalignant colonic cell line, and in 5 of 7 resected colorectal adenocarcinoma cases, compared to adjacent benign colonic epithelium (15). Interestingly, in this study ETH1 expression in benign epithelium showed strong apical (luminal) localization, with lower expression deeper in the lamina, while in colonic adenocarcinoma the expression was higher and uniform (15). Our work herein replicates these findings. Additionally, ETHE1 was over-expressed, roughly 27 -fold, in phenotypically normal colonic epithelial cells from individuals with familial adenomatous polyposis, compared to normal colonic epithelial cells (17). Elevated ETHE1 expression in two colon cancer cell lines also increased aerobic glycolysis (the Warburg effect), oxidative phosphorylation, and mitochondrial biogenesis. Interestingly, higher ETHE1 expression lowered 

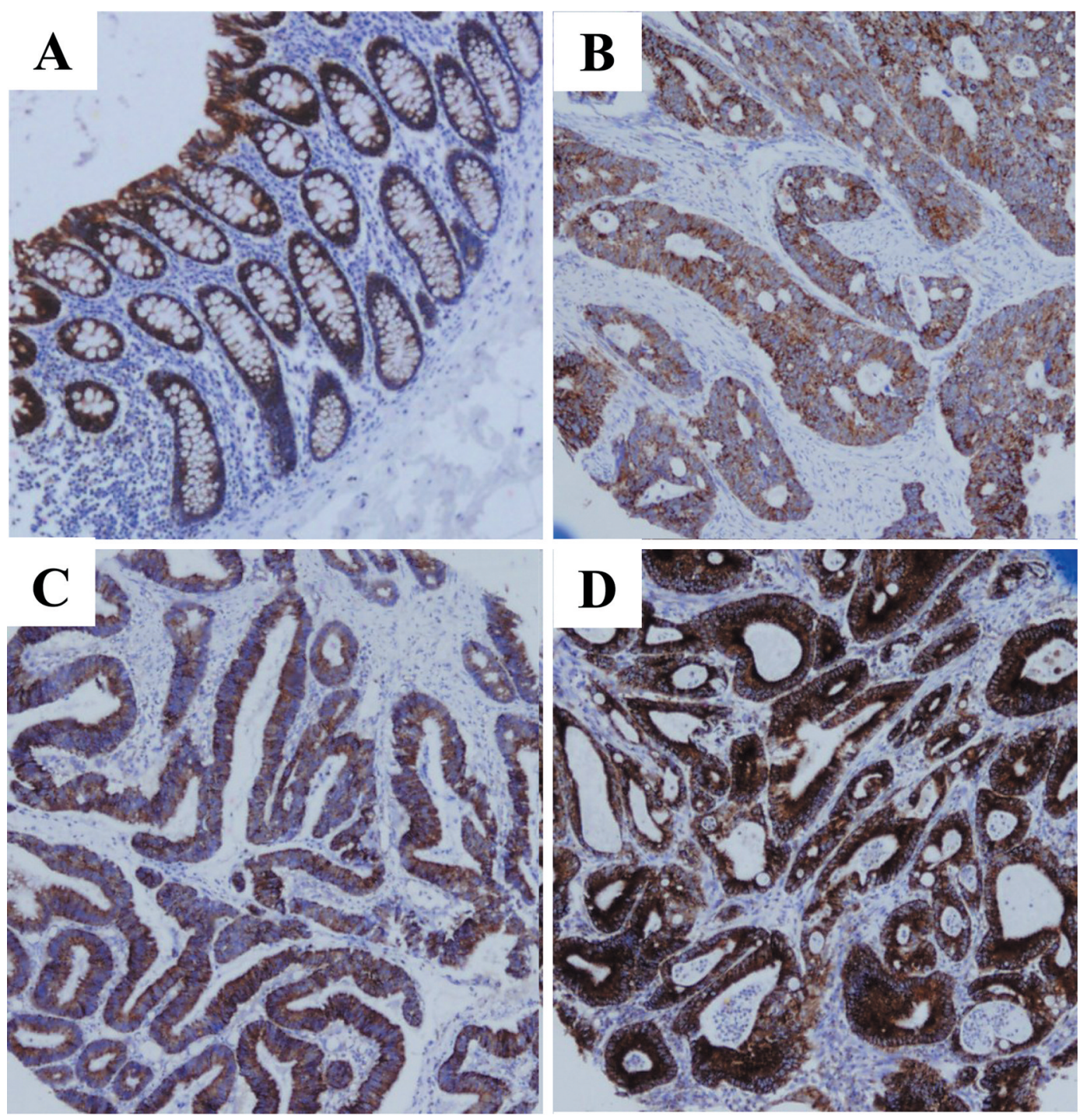

Figure 1. Representative ethylmalonic encephalopathy 1 protein immunostaining of benign colonic epithelium (A), Grade I adenocarcinoma (B), seminoma (C), Grade II adenocarcinoma (D), and (E). Grade III adenocarcinoma. Original magnification $\times 100$.

cellular H2S concentrations (measured by lower glutathione persulfide levels), resulting in increased phosphodiesterase activity (PDE), and adenosine monophosphate-activated protein kinase (AMPKp), sirtuin1 (SIRT1), and peroxisome proliferation-activated receptor $\gamma$-coactivator- $1 \alpha$ activation (PGC1 $\alpha, 18)$. In a murine xenograft model, high ETHE1 expression also accelerated colon cancer cell line growth, with increased Ki-67 and CD31 expression, reflecting increased cell growth with concomitant intensified tumor angiogenesis (18).

Conversely, a comparison of twenty colonic adenocarcinomas to patient-matched benign tissues revealed lower ETHE1 mRNA and protein expression in malignant tissues compared to benign (19). Additionally, a meta-analysis revealed suppressed colonic adenocarcinoma ETHE1 expression compared to benign colonic epithelium. This event was hypothesized to allow the malignant cells to more efficiently produce energy under hypoxic conditions (20).

The reason for these discrepancies may lie in several factors. First, TMA studies do not usually compare patientmatched tissues, while studies that do may show more exact resolution of benign/malignant expression differences. Also, different analytical techniques and sample sizes may give different results. Herein we analyzed a relatively large number of colonic adenocarcinoma samples and found moderate ETHE1 induction compared to benign colonic epithelium. Interestingly, in $38 \%$ of the colon adenocarcinomas examined 
ETHE1 IHC was roughly the same as that seen in benign tissue, with 54\% showing moderately increased expression, and only $18 \%$ showing high ETHE1 expression. Additionally, Libiad et al. (15), found increased ETHE1 expression in 5 of 7 matched benign colon epithelium and matched colonic adenocarcinoma cases, with two cases showing high and equal ETHE1 expression between benign and malignant colonic tissues. Taken together, these data indicate that ETHE1 expression is on average moderately induced in colon adenocarcinoma, with the degree of induction showing some variation. Further support for ETHE1 being increased in colonic malignancy comes for our observation that ETHE1 expression increases with increasing tumor grades.

Polysulfide formation has been implicated in several malignancies. For example, polysulfides are higher in highgrade gliomas compared to glioma-free areas of the brain, while brain hemispheres with glioblastoma show higher polysulfides than the contra-lateral hemispheres without glioblastoma (21, 22). Similarly, surface-enhanced Raman spectroscopy of 186 stage III or IV ovarian cancers indicated that higher CSE expression correlated with increased tumor polysulfides, tumor cisplatin resistance, and a poor prognosis. Cisplatin resistance was found to be related to polysulfide synthesis (23).

Herein we found that ETHE1 expression is increased in colonic adenocarcinomas. Previously ETHE1 was found to catabolize cellular $\mathrm{H} 2 \mathrm{~S}$ while synthesizing polysulfides, and also stimulate aerobic glycolysis, mitochondrial biogenesis, and PDE, AMPKp, and PGC1 $\alpha$ activities, while potentiating tumor growth and angiogenesis $(15,18)$. These data suggest that ETHE1 contributes to colon carcinogenesis, in part, by modulating tumor cell bioenergetics via the conversion of $\mathrm{H} 2 \mathrm{~S}$ into polysulfides, contributing to colon cancer carcinogenesis and growth. Further studies on the roles of polysulfides in cancer and cancer cell biogenetics will be needed to clarify this hypothesis. Lastly, the finding that ETHE1 expression shows a strong apical localization and lower expression in underlying benign colonic epithelium, while colon adenocarcinoma shows strong and uniform ETHE1 expression, suggests that these expression differences may be used in IHC analyses to identify colon cancer in conjunction with histologic analysis and other IHC stains (15).

\section{Conflicts of Interest}

The Authors declare no conflicts of interest.

\section{Authors' Contributions}

All Authors contributed to the writing of the manuscript. Drs. Shackelford and Kevil also performed the literature searches. Dr. Ozluk also took the photographs.

\section{Acknowledgements}

We thank Jennifer Burton for help in the assembling and proof reading of this manuscript. This work was supported by an Institutional Development Award (IDeA) from the National Institutes of General Medical Sciences of the NIH under grant number GM121307 and HL149264 from the National Heart, Lung, and Blood Institute to C.G.K.

\section{References}

1 Mineri R, Rimoldi M, Burlina AB, Koskull S, Perletti C, Heese B, von Döbeln U, Mereghetti P, Di Meo I, Invernizzi F, Zeviani M, Uziel $G$ and Tiranti V: Identification of new mutations in the ETHE1 gene in a cohort of 14 patients presenting with ethylmalonic encephalopathy. J Med Genet 45(7): 473-478, 2008. PMID: 18593870. DOI: 10.1136/jmg.2008.058271

2 Palzkill T: Metallo- $\beta$-lactamase structure and function. Ann N Y Acad Sci 1277: 91-104, 2013. PMID: 23163348. DOI: 10.1111/ j.1749-6632.2012.06796.x

3 Pettinati I, Brem J, McDonough MA and Schofield CJ: Crystal structure of human persulfide dioxygenase: structural basis of ethylmalonic encephalopathy. Hum Mol Genet 24(9): 24582469, 2015. PMID: 25596185. DOI: $10.1093 / \mathrm{hmg} / \mathrm{ddv} 007$

4 Tiranti V, D'Adamo P, Briem E, Ferrari G, Mineri R, Lamantea E, Mandel H, Balestri P, Garcia-Silva MT, Vollmer B, Rinaldo P, Hahn SH, Leonard J, Rahman S, Dionisi-Vici C, Garavaglia $\mathrm{B}$, Gasparini P and Zeviani M: Ethylmalonic encephalopathy is caused by mutations in ETHE1, a gene encoding a mitochondrial matrix protein. Am J Hum Genet 74(2): 239-252, 2004. PMID: 14732903. DOI: $10.1086 / 381653$

5 Tiranti V, Briem E, Lamantea E, Mineri R, Papaleo E, De Gioia L, Forlani F, Rinaldo P, Dickson P, Abu-Libdeh B, CindroHeberle L, Owaidha M, Jack RM, Christensen E, Burlina A and Zeviani M: ETHE1 mutations are specific to ethylmalonic encephalopathy. J Med Genet 43(4): 340-346, 2006. PMID: 16183799. DOI: $10.1136 / j m g .2005 .036210$

6 Hildebrandt TM and Grieshaber MK: Three enzymatic activities catalyze the oxidation of sulfide to thiosulfate in mammalian and invertebrate mitochondria. FEBS J 275(13): 3352-3361, 2008. PMID: 18494801. DOI: 10.1111/j.1742-4658.2008.06482.x

7 Kabil O and Banerjee R: Characterization of patient mutations in human persulfide dioxygenase (ETHE1) involved in H2S catabolism. J Biol Chem 287(53): 44561-44567, 2012. PMID: 23144459. DOI: $10.1074 /$ jbc.M112.407411

8 Jung $\mathrm{M}$, Kasamatsu S, Matsunaga $\mathrm{T}$, Akashi S, Ono K, Nishimura A, Morita M, Abdul Hamid H, Fujii S, Kitamura H, Sawa $\mathrm{T}$, Ida $\mathrm{T}$, Motohashi $\mathrm{H}$ and Akaike $\mathrm{T}$ : Protein polysulfidation-dependent persulfide dioxygenase activity of ethylmalonic encephalopathy protein 1. Biochem Biophys Res Commun 480(2): 180-186, 2016. PMID: 27742479. DOI: $10.1016 / \mathrm{j} . \mathrm{bbrc} .2016 .10 .022$

9 Ida T, Sawa T, Ihara H, Tsuchiya Y, Watanabe Y, Kumagai Y, Suematsu M, Motohashi H, Fujii S, Matsunaga T, Yamamoto M, Ono K, Devarie-Baez NO, Xian M, Fukuto JM and Akaike T: Reactive cysteine persulfides and S-polythiolation regulate oxidative stress and redox signaling. Proc Natl Acad Sci USA 111(21): 7606-7611, 2014. PMID: 24733942. DOI: 10.1073/pnas. 1321232111 
10 Kimura Y, Toyofuku Y, Koike S, Shibuya N, Nagahara N, Lefer D, Ogasawara $\mathrm{Y}$ and Kimura H: Identification of H2S3 and H2S produced by 3 -mercaptopyruvate sulfurtransferase in the brain. Sci Rep 5: 14774, 2015. PMID: 26437775. DOI: 10.1038/srep14774

11 Szabo C, Coletta C, Chao C, Módis K, Szczesny B, Papapetropoulos A and Hellmich MR: Tumor-derived hydrogen sulfide, produced by cystathionine- $\beta$-synthase, stimulates bioenergetics, cell proliferation, and angiogenesis in colon cancer. Proc Natl Acad Sci USA 110(30): 12474-12479, 2013. PMID: 23836652. DOI: 10.1073/pnas.1306241110

12 Honda K, Hishiki T, Yamamoto S, Yamamoto T, Miura N, Kubo A, Itoh M, Chen WY, Takano M, Yoshikawa T, Kasamatsu T, Sonoda S, Yoshizawa H, Nakamura S, Itai Y, Shiota M, Koike D, Naya M, Hayakawa N, Naito Y, Matsuura T, Iwaisako K, Masui T, Uemoto S, Nagashima K, Hashimoto Y, Sakuma T, Matsubara O, Huang W, Ida T, Akaike T, Masugi Y, Sakamoto M, Kato T, Ino Y, Yoshida H, Tsuda H, Hiraoka N, Kabe Y and Suematsu M: On-tissue polysulfide visualization by surface-enhanced Raman spectroscopy benefits patients with ovarian cancer to predict postoperative chemosensitivity. Redox Biol 41: 101926, 2021. PMID: 33752108. DOI: 10.1016/j.redox.2021.101926

13 Meram AT, Chen J, Patel S, Kim DD, Shirley B, Covello P, Coppola D, Wei EX, Ghali G, Kevil CG and Shackelford RE: Hydrogen sulfide is increased in oral squamous cell carcinoma compared to adjacent benign oral mucosae. Anticancer Res 38(7): 3843-3852, 2018. PMID: 29970504. DOI: 10.21873/anticanres. 12668

14 Turbat-Herrera EA, Kilpatrick MJ, Chen J, Meram AT, Cotelingam J, Ghali G, Kevil CG, Coppola D and Shackelford RE: Cystathione $\beta$-synthase is increased in thyroid malignancies. Anticancer Res 38(11): 6085-6090, 2018. PMID: 30396922. DOI: 10.21873 /anticanres.12958

15 Libiad M, Vitvitsky V, Bostelaar T, Bak DW, Lee HJ, Sakamoto N, Fearon E, Lyssiotis CA, Weerapana E and Banerjee R: Hydrogen sulfide perturbs mitochondrial bioenergetics and triggers metabolic reprogramming in colon cells. J Biol Chem 294(32): 12077-12090, 2019. PMID: 31213529. DOI: 10.1074/jbc.RA119.009442

16 Pan J, Song G, Chen D, Li Y, Liu S, Hu S, Rosa C, Eichinger D, Pino I, Zhu H, Qian J and Huang Y: Identification of serological biomarkers for early diagnosis of lung cancer using a protein array-based approach. Mol Cell Proteomics 16(12): 2069-2078, 2017. PMID: 29021294. DOI: 10.1074/mcp.RA117.000212

17 Yeung AT, Patel BB, Li XM, Seeholzer SH, Coudry RA, Cooper HS, Bellacosa A, Boman BM, Zhang T, Litwin S, Ross EA, Conrad P, Crowell JA, Kopelovich L and Knudson A: One-hit effects in cancer: altered proteome of morphologically normal colon crypts in familial adenomatous polyposis. Cancer Res 68(18): 7579-7586, 2008. PMID: 18794146. DOI: 10.1158/00085472.CAN-08-0856
18 Witherspoon M, Sandu D, Lu C, Wang K, Edwards R, Yeung A, Gelincik O, Manfredi G, Gross S, Kopelovich L and Lipkin S: ETHE1 overexpression promotes SIRT1 and PGC1 $\alpha$ mediated aerobic glycolysis, oxidative phosphorylation, mitochondrial biogenesis and colorectal cancer. Oncotarget 10(40): 4004-4017, 2019. PMID: 31258845. DOI: 10.18632/oncotarget.26958

19 Zhang S, Jin J, Tian X and Wu L: hsa-miR-29c-3p regulates biological function of colorectal cancer by targeting SPARC. Oncotarget 8(61): 104508-104524, 2017. PMID: 29262657. DOI: $10.18632 /$ oncotarget.22356

20 Piran M, Sepahi N, Moattari A, Rahimi A and Ghanbariasad A: Systems biomedicine of primary and metastatic colorectal cancer reveals potential therapeutic targets. Front Oncol 11: 597536, 2021. PMID: 34249670. DOI: 10.3389/fonc.2021.597536

21 Wróbel M, Czubak J, Bronowicka-Adamska P, Jurkowska H, Adamek D and Papla B: Is development of high-grade gliomas sulfur-dependent? Molecules 19(12): 21350-21362, 2014. PMID: 25532835. DOI: 10.3390/molecules 191221350

22 Shiota M, Naya M, Yamamoto T, Hishiki T, Tani T, Takahashi H, Kubo A, Koike D, Itoh M, Ohmura M, Kabe Y, Sugiura Y, Hiraoka N, Morikawa T, Takubo K, Suina K, Nagashima H, Sampetrean O, Nagano O, Saya H, Yamazoe S, Watanabe H and Suematsu M: Gold-nanofève surface-enhanced Raman spectroscopy visualizes hypotaurine as a robust anti-oxidant consumed in cancer survival. Nat Commun 9(1): 1561, 2018. PMID: 29674746. DOI: 10.1038/s41467-018-03899-1

23 Honda K, Hishiki T, Yamamoto S, Yamamoto T, Miura N, Kubo A, Itoh M, Chen WY, Takano M, Yoshikawa T, Kasamatsu T, Sonoda S, Yoshizawa H, Nakamura S, Itai Y, Shiota M, Koike D, Naya M, Hayakawa N, Naito Y, Matsuura T, Iwaisako K, Masui T, Uemoto S, Nagashima K, Hashimoto Y, Sakuma T, Matsubara O, Huang W, Ida T, Akaike T, Masugi Y, Sakamoto M, Kato T, Ino Y, Yoshida H, Tsuda H, Hiraoka N, Kabe Y and Suematsu M: Ontissue polysulfide visualization by surface-enhanced Raman spectroscopy benefits patients with ovarian cancer to predict postoperative chemosensitivity. Redox Biol 41: 101926, 2021. PMID: 33752108. DOI: 10.1016/j.redox.2021.101926
Received August 6, 2021

Revised September 1, 2021

Accepted September 14, 2021 